Article

\title{
The Balance Strategy between Structural Safety and Sensing Accuracy Inspired by Slit-Based Mechanical Sensilla
}

\author{
Qian Wang ${ }^{\dagger}$, Cheng Fan ${ }^{\dagger}$, Yuecheng Gui, Yao Lu and Kejun Wang * \\ Jiangsu Provincial Key Laboratory of Advanced Robotics, Soochow University, Suzhou 215123, China; \\ qianwang@suda.edu.cn (Q.W.); chfan@suda.edu.cn (C.F.); 20195229009@stu.suda.edu.cn (Y.G.); \\ 20185229072@stu.suda.edu.cn (Y.L.) \\ * Correspondence: kjwang@suda.edu.cn \\ + Qian Wang and Cheng Fan have contributed equally to this work.
}

Received: 8 November 2020; Accepted: 4 December 2020; Published: 8 December 2020

\begin{abstract}
In engineering, cracks are typically regarded as defects due to enormous stress amplification at tip of the crack. Conversely, scorpion ingeniously utilizes the "risky" near-tip stress field of a crack-shaped slit to accurately detect weak vibration signal without causing catastrophic crack propagation from the slit tip. The present paper focuses on the balance strategy between structural safety and sensing accuracy of slit-based mechanical sensilla. We performed a detailed structural and mechanical property study of tissue around the slit wake utilizing a complementary combination of various experimental methods. The results indicate that there is a special thin surface membrane covering the slit wake and the elastic moduli of the membrane and exoskeleton are $0.562 \mathrm{GPa}$ and $5.829 \mathrm{GPa}$, respectively. In addition, the ratio of bending stiffness between exoskeleton and membrane tissue is about $8 \times 10^{4}$. The theoretical and simulation analysis show that the surface membrane-with appropriate elastic modulus and bending stiffness - can achieve different forms of deformation with the change of slit width for protecting the mechanosensory structure without sacrificing the sensing accuracy. This finding offers a crucial theoretical basis for the further design of bionic mechanical sensors based on the near-tip stress field of artificial cracks.
\end{abstract}

Keywords: crack; mechanosensory structure; structural safety; sensing accuracy; balance

\section{Introduction}

Bionic engineering provides a promising alternative method in expediting the development of artificial systems [1-3]. Recently, researchers have been revealing many ingenious sensing strategies of animals and plants, thereby providing ample inspiration for the next-generation artificial sensors [4-7]. In various types of signals, the perception of mechanical signals, such as vibration, sound, air/water flow and tactile, is crucial for the survival of animals with highly degraded visual systems [8-10]. The corresponding mechanical sensilla, composed of mechanosensory microstructure, special materials and sensory neurons, have provided broad biologically inspired strategies for designing mechanical sensors with high performance [11-15]. In nature, mechanical signals can only be sensed by organisms after inducing the deformation of specialized mechanosensory structure, which requires that the excellent sensing ability must be based on the outstanding resistance to catastrophic fatigue failure of sensory materials [16-18]. Therefore, revealing the structural stability mechanism of mechanical sensilla is one of particularly importance in developing bionic mechanical sensors.

In various kinds of mechanical sensilla, the slit-based vibrational sensilla of arachnids (spiders, scorpions, etc.), which are composed of crack-shaped slits with different lengths that can detect very weak vibration signals from several decimeters or even meters away, are worth noticing [19,20]. As a 
mechanosensory structure, a slit is embedded in each walking leg, and the wake of the slit opens and closes alternately under cyclic loads caused by walking and the substrate vibration signal [21]. The scorpion is an arachnid, whose Basitarsal Compound Slit Sensilla (BCSS) composed of slit arrays has been investigated extensively with respect to its morphology and functional mechanism [20,22]. In particular, research on the excellent sensing mechanism of the slit unit indicates that to maximize the sensitivity of BCSS, the scorpion has evolved an ingenious strategy to make a "risky" nanoscale near-tip stress field, and the receptive field of mechanosensory neurons overlap at a crack-shaped slit tip [9]. The near-tip stress field is responsible for the highly efficient collection of dispersed mechanical signals which are converted into electrical signals through sensory neurons. Nevertheless, large stress amplification at a crack tip usually leads to crack propagation which creates enormous risks for engineered structures [23,24]. Obviously, the generation of a crack from its flaw-like tip will make a slit completely lose the function of mechanical signal detection. Hence, the crack-shaped slit as a sensory structure should not only generate sufficiently large stress amplification for excellent perceptive function but will also have superior crack resistance for avoiding catastrophic fractures from the slit tip. However, while the outstanding sensing mechanism of slit-based mechanical sensilla has been revealed recently, there is still a poor understanding of how excellent fracture toughness is achieved without sacrificing high accuracy.

Herein, the structural mechanism and perceptual ability of the BCSS was comprehensively investigated through experimental and modeling approaches, including scanning electron microscopy, semi-thin sectioning, nanoindentation testing and finite element modeling. As a result, this work provides a crucial biomimetic design strategy for the further development of mechanical sensors utilizing the near-tip stress field.

\section{Experimental}

\section{Specimens}

The scorpions Heterometrus petersii, collected from the Hainan province of China, were kept in a laboratory which simulated the natural environment. All scorpion treatments complied with Chinese law regarding the protection of animals.

\section{The Characterization of Morphology and Microstructure}

The morphology of the tissue around mechanical sensilla was obtained by stereoscopic microscopy (VHX-5000, KEYENCE, Osaka Prefecture, Japan). The internal structural characteristics of fixed tissue were obtained through a field emission SEM (JSM-6700F, JEOL, Akishima City, Tokyo, Japan).

\section{Nano-Indentation}

Nano-indentation characterizations of biological tissue around a slit wake were conducted utilizing a nanoindentation system (Nanoindenter G200, Agilent Technologies Inc., Santa Clara, CA, USA) with indentation depths of $2000 \mathrm{~nm}$. The elastic modulus of tissue was calculated based on the load-displacement curves and the standard Oliver-Pharr and Suresh methods [25]. For sample preparation, a liquid cyanoacrylate thin film (about $1000 \mathrm{~nm}$ ), which can cure quickly after contacting with vapor, was first spun coated on a glass substrate. Next, the prepared tissue was quickly placed on the cyanoacrylate thin film before thorough curing of the cyanoacrylate. In the whole process, a light microscope which was used to confirm the outer surface of biological tissue was oriented parallel to the object stage and was not polluted by cyanoacrylate.

\section{Atomic Force Microscopy Imaging (AFM)}

Determinations of surface morphology and elastic modulus were performed using a Dimension Icon AFM system (Bruker Nano, Santa Barbara, CA, USA). The scanning mode based on Quantitative Nanomechanical Mapping (QNM) and Peak Force QNM was used in this experiment. The probe with a spring constant of $0.7 \mathrm{~N} / \mathrm{m}$ was utilized. The process of sample preparation was the same as that for nano-indentation. 


\section{Results and Discussion}

The scorpion $H$. petersii has a slit-based mechanical sensilla which is located at the basitarsus of each walking leg and consists of 12 curved slits (Figure 1a-c). The length of slits decreases from about $320 \mu \mathrm{m}$ to $50 \mu \mathrm{m}$. Transversal cross-section of the BCSS was imaged through histological section and toluidine blue staining. The results indicate that each slit connected with the dendrite of sensory neurons through a coupling cylinder at the slit tip (Figure 1d). Longitudinal cross-sections through the slit were imaged by semithin section and SEM, which revealed that both sides of the slit wake were almost completely disconnected in the direction of exoskeleton thickness (Figure 1e). In addition, magnified views of the longitudinal cross-section further show that there is special surface membrane tissue covering the wake of the slit (Figure 1f). Figure 1f clearly indicates that there is obvious bending deformation of the surface membrane. The periodic closure and opening of the microscale slit under cyclic loads caused by walking and vibration signals was discovered in a previous study [9]. Hence, the surface membrane covering the slit wake may fold periodically under alternating loads. The deformation mode as well as the function of the surface membrane will be further discussed in detail in the following theoretical and simulation analysis. The statistical data of detailed structural parameters indicates that the initial width $\left(w_{0}\right)$ of the slit is about $8 \mu \mathrm{m}$, the thickness $\left(t_{e}\right)$ of hard exoskeleton is about $50 \mu \mathrm{m}$, the thickness $\left(t_{m}\right)$ of the membrane is about $2.5 \mu \mathrm{m}$, and the interval $\left(d_{0}\right)$ between adjacent slits increases from $24 \mu \mathrm{m}$ to $120 \mu \mathrm{m}$. The symbols for sensilla geometrical dimensions are shown in Figure 2a. In summary, the above study on structural characterization clearly indicates that mechanosensory slits are local micro-cracks embedded in the hard exoskeleton, and the wake of the slit is covered by a special surface membrane. From the perspective of engineering, the slits are risky cracks embedded in the stress body.

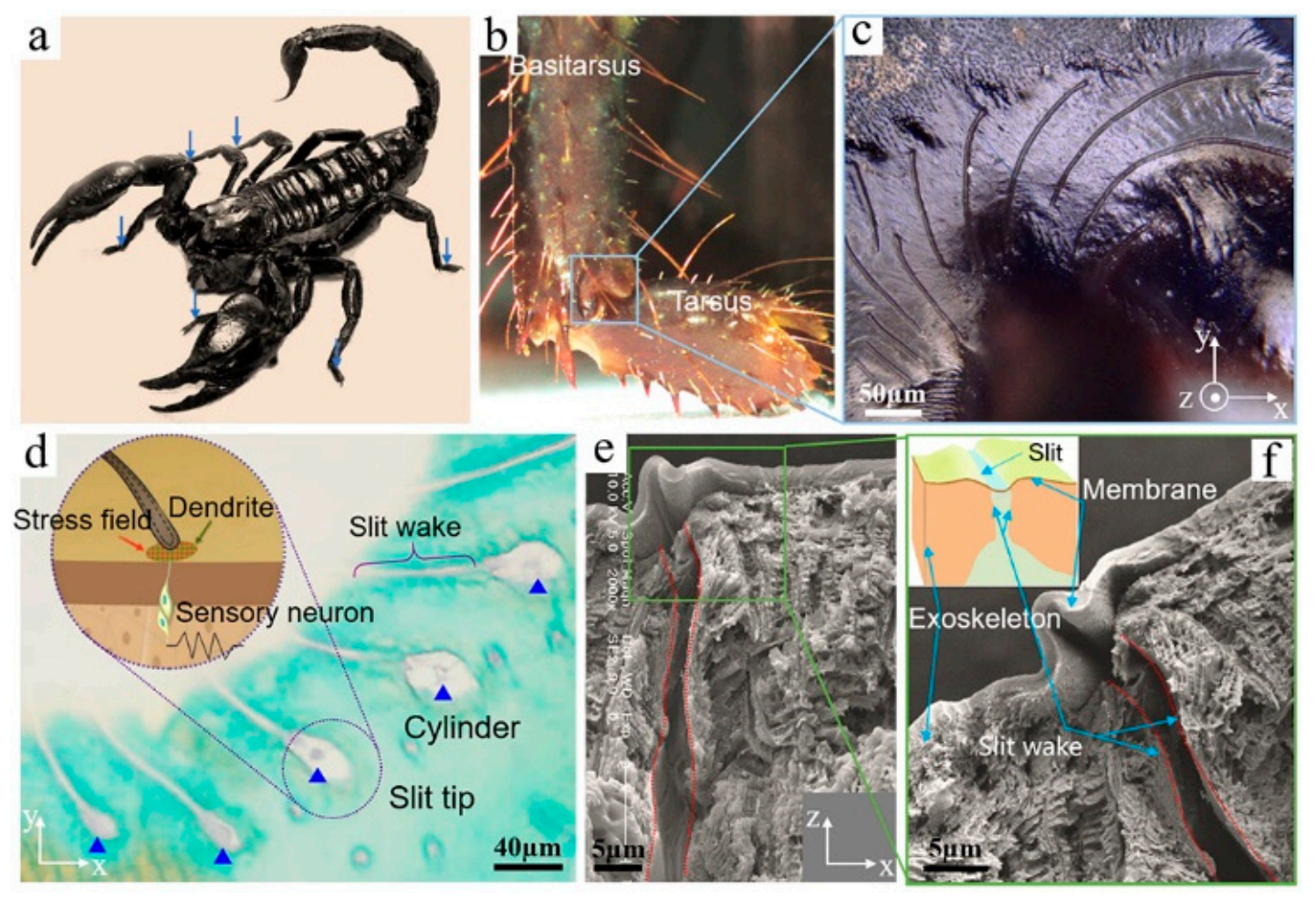

Figure 1. (a) The adult scorpion H. petersii collected from Hainan Province, China; (b) the distribution of the BCSS on eight walking legs; (c) the morphology of Basitarsal Compound Slit Sensilla (BCSS) with slits arranged in fan shape; (d) transversal cross-section with toluidine blue staining; (e) longitudinal cross-section image; (f) local enlarged image taken from (e). 


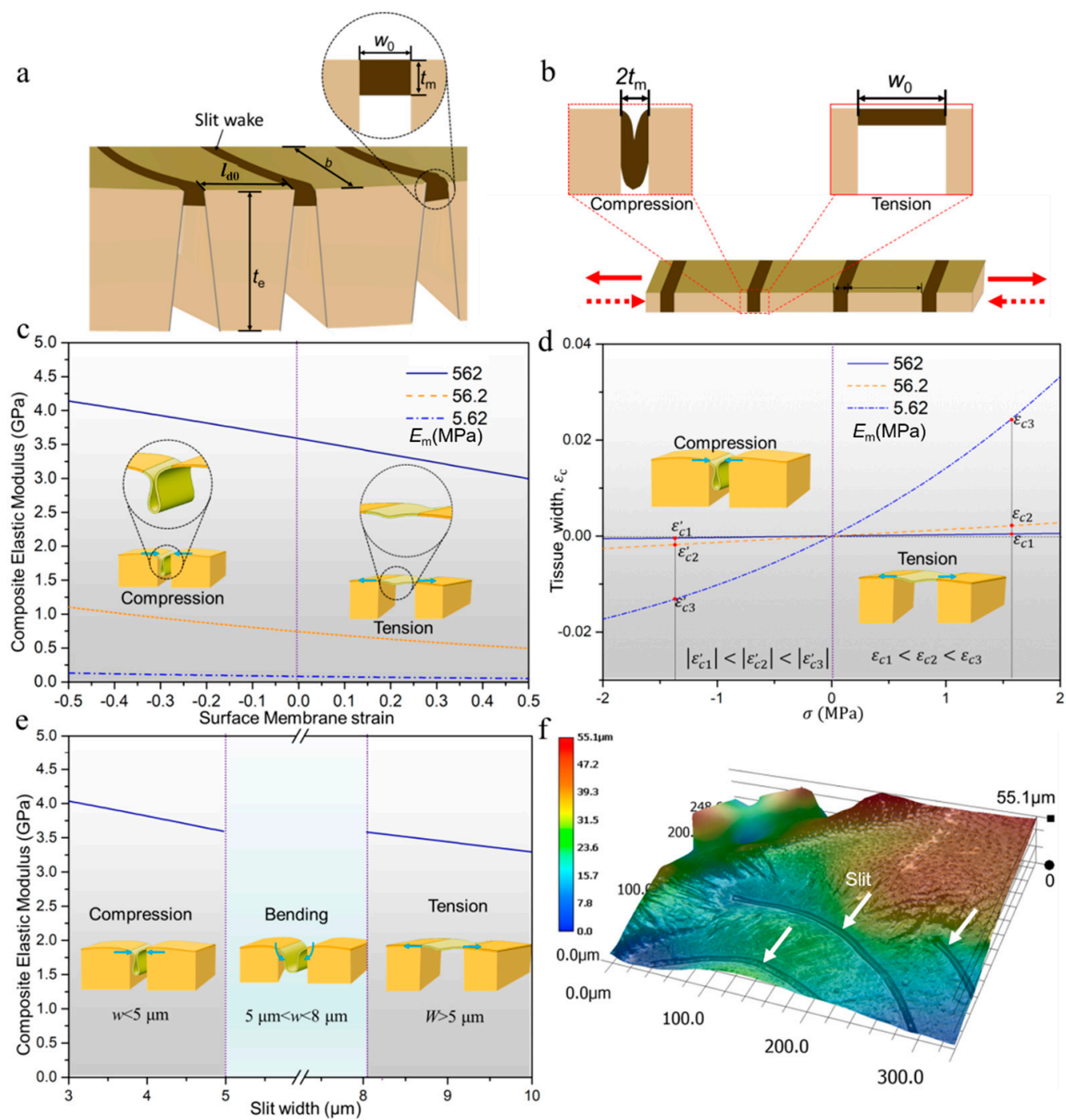

Figure 2. (a) Structural model of tissue around a slit wake; (b) simplified structural model based on (a) for analyzing the process of tension and compression; (c) the composite elastic modulus as a function of membrane strain under different values of $E m$; (d) the relationship between the strain of composite tissue and applied stress under different values of $E m ;(\mathbf{e})$ the deformation type of surface membrane in different range of slit width; (f) three-dimensional surface morphology of tissue around a slit wake.

The unique mechanical behavior of biological materials is an important factor for revealing the role of the surface membrane in balancing perception accuracy and structural safety of slit-based mechanosensory structure. Hence, we further analyzed the elastic modulus of fresh biological tissue around the slit wake through the AFM and nano-indenter. Figure $3 a$ illustrates that, under fresh conditions, the elastic modulus of the surface membrane is strikingly lower than the exoskeleton. In contrast, the difference on the elastic modulus between them is not obvious in a dehydrated state (Figure 3b). In fact, this difference on tissue elastic modulus for different physiological states is mainly caused by water, which has also been reported for other biological materials [26]. The difference on the elastic modulus of membrane and exoskeleton further indicates that the flexible membrane with relatively low stiffness is more prone to deformation than the hard exoskeleton with the opening and closure of a slit. For additional measurements of an accurate value of elastic modulus on these two kinds of tissue, the nano-indentation experiment was carried out (Figure 3c). The result indicates that the elastic modulus of surface membrane $\left(E_{m}\right)$ and exoskeleton $\left(E_{s}\right)$ were $0.562 \mathrm{GPa}$ and $5.829 \mathrm{GPa}$, respectively (Figure 3d). 


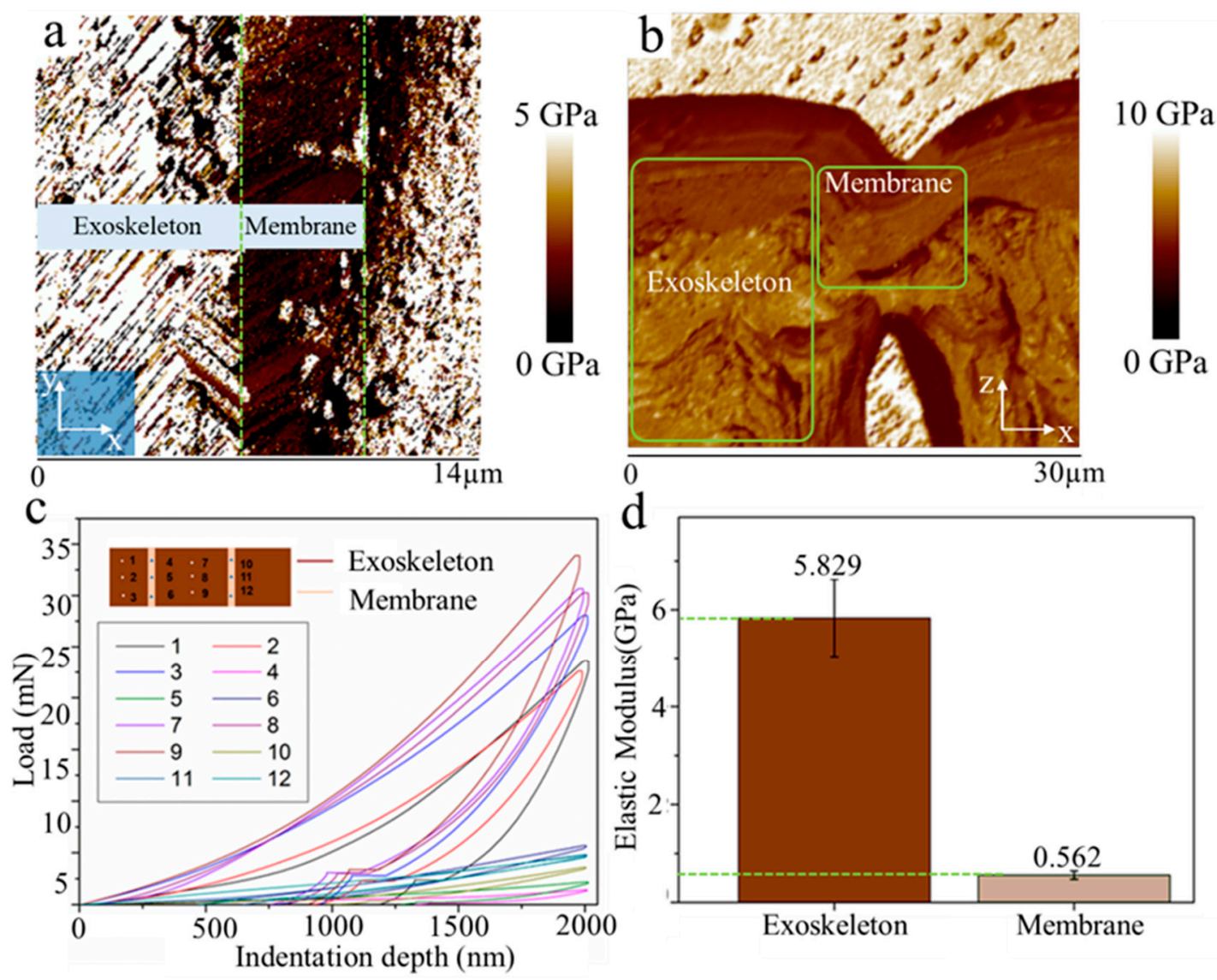

Figure 3. (a) Elastic modulus map of tissue around a slit wake under fresh conditions; (b) elastic modulus map of tissue in dehydrated state; (c) nano-indentation load displacement curves of membrane tissue and exoskeleton, respectively; (d) the elastic modulus of exoskeleton and membrane tissue calculated based on the curves in (c).

Based on the microstructural characteristics and mechanical performance of tissue around the slit wake, we further analyzed the balancing strategy of sensing accuracy and structural safety. Fracture mechanics studies have proven that the pre-existing crack will further propagate from the near-tip stress field with the excessive opening and closure of crack. Longitudinal cross-section image indicates that the tissue around slit wake can be regarded as a multi-phase laminated tissue (Figure 1e). The composite elastic modulus can effectively represent the ability of multi-phase laminated tissue to resist tensile and compressive deformation. Hence, we theoretically analyzed the composite elastic modulus $\left(E_{c}\right)$ of tissue around a slit wake, which can easily predict the ability of the surface membrane to control the opening displacement (i.e., the width) of the slit and then further avoid new crack nucleation from the slit tip. Firstly, the multi-phase laminated structural model approximating the longitudinal section morphology is given in Figure 2a, which clearly indicates that the membrane is sandwiched between the exoskeleton. In tensile and compressive deformation of surface membranes, this structural model can be further simplified to equal the thickness model in Figure $2 b$, because the vertical height of the effective stress surface is equal to the thickness of the membrane. The composite elastic modulus $\left(E_{c}\right)$ for this typical tessellated structural model in Figure $2 \mathrm{~b}$ is calculated through an isostress description of composite behavior and given by [27]:

$$
E_{c}=\left[\frac{f_{m}}{E_{m}}+\frac{\left(1-f_{m}\right)}{E_{s}}\right]^{-1}=\frac{E_{s}}{1+\left(\frac{E_{s}}{E_{m}}-1\right) f_{m}}
$$


where $f_{m}$ is the volume fraction of membrane tissue in the tessellated structural model (Figure $2 \mathrm{~b}$ ). The $f_{m}$ would be changed when there is stress acting on the sensilla. Hence, the $f_{m}$ can be further given by:

$$
f_{m}=\frac{l_{m}}{\left(l_{m}+l_{d}\right)}
$$

here, $l_{m}$ and $l_{d}$ are represent the width of membrane tissue and exoskeleton, respectively.

The strain, $\varepsilon_{m}$, of the membrane tissue located at the slit wake can be given by:

$$
\varepsilon_{m}=\int_{l_{m 0}}^{l_{m}} \frac{d l}{l}=\ln \left(\frac{l_{m}}{l_{m 0}}\right)
$$

here, $l_{m 0}$ is the initial width of the membrane tissue. With the mechanical signal acting on the BCSS, the membrane tissue would experience three types of stress (i.e., tensile stress, bending stress and compressive stress), successively. At initial stage of tension, $l_{m 0}$ can be represented by the original width, $w_{0}$, of a crack-shaped slit. At the initial stage of compression, $l_{m 0}$ is two times the thickness, $t_{m}$, of the membrane tissue, because of the folding deformation at the bending stage (Figure $2 b$ ). In summary, the initial width, $l_{m 0}$, of membrane tissue can be given:

$$
l_{m 0}=\left\{\begin{array}{rr}
w_{0}, & \text { tension } \\
2 \mathrm{t}_{m}, & \text { compression }
\end{array}\right.
$$

At the stage of bending deformation, the $\varepsilon_{m}$ of membrane tissue can be approximately regarded as zero, because the stress acting on the membrane is extremely small. Hence, the $\varepsilon_{m}$ of membrane tissue can be further given by:

$$
\varepsilon_{m}=\left\{\begin{array}{rr}
\ln \left(\frac{l_{m}}{w_{0}}\right), & \text { tension } \\
0, & \text { bending } \\
\ln \left(\frac{l_{m}}{2 t_{m}}\right), & \text { compression }
\end{array}\right.
$$

In addition, with the change of slit width, the strain, $\varepsilon_{m}$, of membrane tissue can be further given by:

$$
\varepsilon_{m}=\left\{\begin{aligned}
\ln \left(\frac{w}{l_{m 0}}\right), & w<2 t_{m} \text { or } w>\mathrm{w}_{0} \\
0, & 2 \mathrm{t}_{m} \leq w \leq \mathrm{w}_{0}
\end{aligned}\right.
$$

here, $w$ represents the width of the slit. Similarly, in the tensile or compressive stage, the strain, $\varepsilon_{d}$, of the exoskeleton is given by:

$$
\varepsilon_{d=} \ln \left(\frac{l_{d}}{l_{d 0}}\right)
$$

where $l_{d}$ and $d_{0}$ represent the final and initial slit widths, respectively. Based on the above analysis, Equation (2) can be given by:

$$
f_{m}=\frac{l_{m 0} \exp \left(\varepsilon_{m}\right)}{l_{m 0} \exp \left(\varepsilon_{m}\right)+l_{d 0} \exp \left(\varepsilon_{d}\right)}=\left[1+\gamma \exp \left(\frac{\sigma_{s i g}}{E_{s}}-\frac{\sigma_{s i g}}{E_{m}}\right)\right]^{-1}
$$

here, the applied stress $\sigma_{s i g}$ acting on the membrane and the epicuticle is given by: $\sigma_{s i g}=E_{m} \varepsilon_{m}=E_{s} \varepsilon_{d}$, and $\gamma=d_{0} / l_{m 0}$. In addition, $f_{m}$ can be represented by:

$$
f_{m}=\frac{l_{m 0} \exp \left(\varepsilon_{m}\right)}{l_{m 0} \exp \left(\varepsilon_{m}\right)+l_{d 0} \exp \left(\varepsilon_{d}\right)}=\left\{1+\gamma \exp \left[\varepsilon_{m}\left(\frac{E_{m}}{E_{s}}-1\right)\right]\right\}^{-1}
$$


Substituting Equation (9) into Equation (1), the composite elastic modulus, $E_{c}$, is then given by:

$$
E_{c}=\frac{E_{s}}{1+\left(\frac{E_{s}}{E_{m}}-1\right)\left\{1+\gamma \exp \left[\varepsilon_{m}\left(\frac{E_{m}}{E_{s}}-1\right)\right]\right\}^{-1}}
$$

Based on Equation (10), the $E_{\mathrm{c}}$ as a function of $\varepsilon_{m}$, as well as for different values of $E_{m}$, is plotted in Figure 2c. The result indicates that there is a close relationship between $E_{\mathrm{c}}$ and $E_{\mathrm{m}}$. Specifically, under the same membrane strain, $E_{\mathrm{c}}$ decreases significantly for a decrease in $E_{m}$ value $562 \mathrm{MPa}$ to $5.62 \mathrm{MPa}$. In addition, the strain, $\varepsilon_{\mathcal{C}}$, of composite tissue as a function of applied stress, $\sigma_{\text {sig }}$, can be given by $\varepsilon_{c}=\sigma_{s i g} / E_{c}$. Figure $2 \mathrm{~d}$ shows that, under the same $\sigma_{\text {sig }}$, the absolute value of $\varepsilon_{c}$ is the smallest when $E_{m}$ is equal to the measured value. The above comparative results clearly show that the composite tissue around the slit wake can efficiently absorb strain energy through small strain, $\varepsilon_{\mathcal{C}}$, for the reasonable configuration of $E_{m}$. The membrane strain $\varepsilon_{m}$ as a function of composite $\varepsilon_{c}$ is [28]:

$$
\varepsilon_{m}=\varepsilon_{c}\left(l_{0}+l_{d 0}\right) / l_{0}
$$

where, $l_{0}$ is $w_{0}$ and $2 t_{m}$, respectively, at the initial stage of tension and compression. The $\varepsilon_{m}$ as a function of slit width, $w$, can be given by: $\varepsilon_{m}=\left(\omega-l_{0}\right) / l_{0}$. Therefore, the width of the slit can be further expressed as:

$$
w=l_{0}+\varepsilon_{c}\left(l_{0}+l_{d 0}\right)
$$

Combining Equation (12) and relationship between $\varepsilon_{c}$ and $E_{m}$, it is clear that the change of slit width mainly depends on the mechanical property of the membrane. Specifically, in tensile or compressive stage, the surface membrane can reasonably control slit width through absorbing strain energy with small deformation. The crack-shaped slit will propagate from the slit tip with the excessive opening and closure of the slit wake because of the high stress intensity at the slit tip [29,30]. Therefore, the surface membrane must protect the pre-existing mechanosensory slit through controlling the slit width within a safe threshold.

While the above studies only reveal the protective mechanism of the surface membrane, there is still no reasonable interpretation of how the membrane avoids negative effects on sensing accuracy when a weak vibration signal acts on the BCSS. Hence, we further determined the functional relationship between $E_{c}$ and slit width $w$ based on Equations (10)-(12):

$$
E_{C}= \begin{cases}\frac{E_{s}}{1+\left(\frac{E_{s}}{E_{m}}-1\right)\left[1+\gamma\left(\frac{w}{2 t_{m}}\right)^{\left(\frac{E_{m}}{E_{s}}-1\right)}\right]^{-1}}, & w<2 t_{m} \\ \frac{E_{s}}{1+\left(\frac{E_{s}}{E_{m}}-1\right)\left[1+\gamma\left(\frac{w}{w 0}\right)^{\left(\frac{E_{m}}{E_{s}}-1\right)}\right]^{-1}}, & w>\mathrm{w}_{0}\end{cases}
$$

The $E_{c}$ as a function of $w$ based on Equation (13) is plotted in Figure 2e. The result indicates that, consistent with the finding in Figure 1f, there is bending deformation occurring in the membrane tissue during the signal detection process (i.e., $5 \mu \mathrm{m}<\omega<8 \mu \mathrm{m}$ ). The bending stiffness, which can effectively represent the ability of material to resist bending deformation, is given by

$$
E I=E b t^{3}
$$

where $E I, E, b, t$ are the bending stiffness, elastic modulus, width and thickness of the component, respectively. Figure 2a shows that the width $(b)$ of the exoskeleton and surface membrane are equal in the structural model. Therefore, the ratio $(H)$ of exoskeleton bending stiffness $\left(E_{s} I_{s}\right)$ and membrane bending stiffness $\left(E_{m} I_{m}\right)$ can be presented as $H=E_{s} t_{e}^{3} / E_{m} t_{m}^{3}$. The calculation result indicates that $H \approx 8 \times 10^{4}$, which futher clearly shows that the membrane tissue is more prone to bending deformation than the exoskeleton if the width of the slit is in the range of $5 \mu \mathrm{m}<\omega<8 \mu \mathrm{m}$. Furthermore, Figure $2 \mathrm{f}$ shows that the three-dimensional morphology of the slit wake is typically concave which is easily 
subjected to a bending moment. Under the action of the bending moment, the surface membrane, with extremely small bending stiffness, undergoes bending deformation in the process of signal detection, which does not influence the sensing performance of the slit unit.

In order to further verify the functional mechanism of the surface membrane, we performed finite element simulations based on ANSYS software. The 3D finite element model of a substrate and film is shown in Figure 4a. The crack was modeled at the edge of the substrate. The wake of crack is covered by a layer of polyester film. Similar to the biological material around the slit wake, the elastic modulus ratio of the film and the substrate in this model is about 0.1. In the finite element analysis, constraints are imposed on the left end of the substrate, and a tensile or compressive load is applied on the other end for analyzing the change of stress intensity at the film surface and the tip of the crack. The stress concentration coefficient [31], as a parameter reflecting the degree of local stress increase, was used to characterize the influence of surface membrane on the stress intensity at the slit tip. Figure $4 \mathrm{~b}$ shows that the surface membrane is compressed, bended and stretched, respectively, with the change of slit width, which is in good agreement with the results of the present experiments and theoretical analysis. Meanwhile, the stress concentration coefficient decreases significantly at the stages of tension and compression, but remains constant in the bending stage. In the absence of the membrane, the stress concentration coefficient keeps constant with the change of slit width (Figure $4 \mathrm{~b}$ ), which is consistent with the bending deformation stage of the membrane. The above results further indicate that the stress intensity at the crack tip is only restrained by the surface membrane under compression and tension. In addition, the influence of $E_{m}$ on the stress intensity at the crack tip was analyzed. Figure $4 \mathrm{c}$ shows that the membrane with large elastic modulus has better performance in restraining the stress intensity at a near-tip stress field in the stages of tension and compression. Conversely, the stress concentration coefficient under different membrane moduli does not change significantly in the bending stage, because, as shown in Equation (14), the bending stiffness is mainly determined by the membrane thickness. Hence, the present simulation results are in good agreement with the theoretical analysis, which shows that the surface membrane can achieve an effective balance between sensing accuracy and structural safety through adjusting the type of deformation with the change of signal intensity.
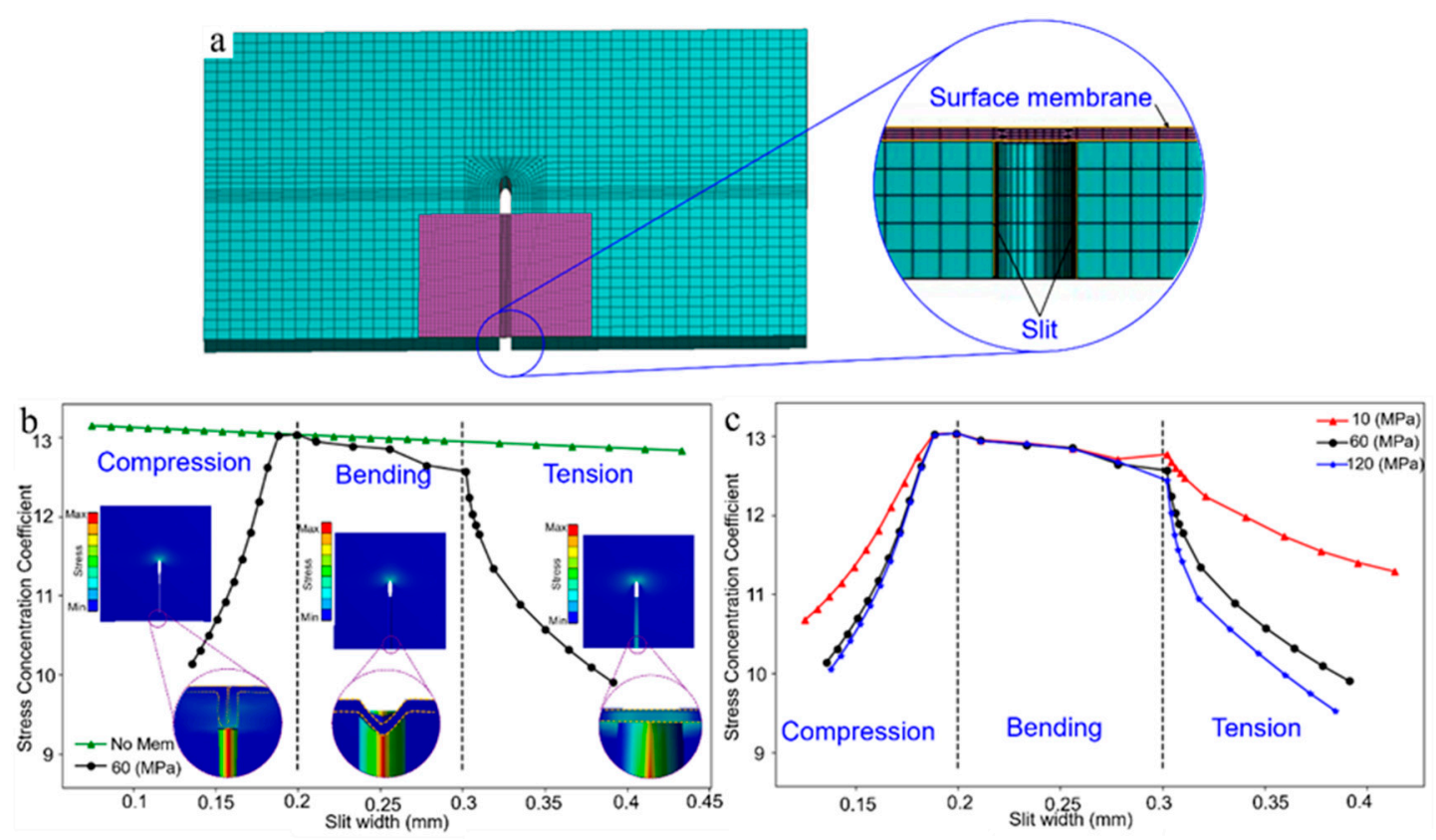

Figure 4. (a) Finite element model mesh; (b) the curve of stress concentration coefficient as a function of crack width; (c) the variation curve of stress concentration coefficient with crack width for different membrane elastic moduli. 


\section{Conclusions}

The mechanism of scorpions utilizing the "risky" near-tip stress field of a slit for sensing was revealed through experimental measurement, theoretical analysis and numerical simulation. The results indicate that, on the one hand, the surface membrane covering the slit wake can absorb strain energy through small deformations that keep the stress intensity at the slit tip within a safe threshold. On the other hand, the bending deformation of the membrane tissue is extremely effective in the process of signal detection for avoiding a negative impact on sensing accuracy. Further work should verify the biological strategy of bionic sensors. Our study provides important new insights for manufacturing an artificial mechanical sensor with a stable structure based on a near-tip slit stress field.

Author Contributions: K.W. conceived of the presented idea. Q.W. and C.F. developed the theory and performed the computations. Y.G. and Y.L. verified the analytical methods by finite element. All authors discussed the results and contributed to the final manuscript. All authors have read and agreed to the published version of the manuscript.

Funding: This research was funded by the National Natural Science Foundation of China (Grant No. 52005356;52005355) and Chinese Postdoctoral Science Fouation (Grant No. 2020T130457).

Conflicts of Interest: The authors declare that they have no conflict of interest.

\section{References}

1. Jung, Y.H.; Park, B.; Kim, J.U.; Kim, T. Bioinspired electronics for artificial sensory systems. Adv. Funct. Mater. 2018, 31, e1803637. [CrossRef] [PubMed]

2. Ma, Y.; Wu, Q.; Duanmu, L.; Wu, S.; Liu, Q.; Li, B.; Zhou, X. Bioinspired composites reinforced with ordered steel fibers produced via a magnetically assisted 3D printing process. J. Mater. Sci. 2020, 55, 15510-15522. [CrossRef]

3. Sun, J.; Wu, W.; Tong, J. Investigating the nanomechanical properties and reversible color change properties of the beetle Dynastes tityus. J. Mater. Sci. 2017, 52, 6150-6160. [CrossRef]

4. Nakata, T.; Phillips, N.; Simoes, P.; Russell, L.J.; Cheney, J.A.; Walker, S.M.; Bomphrey, R.J. Aerodynamic imaging by mosquitoes inspires a surface detector for autonomous flying vehicles. Science 2020, 368, 634-637. [CrossRef]

5. Kuntzman, M.L.; Hall, N.A. Sound source localization inspired by the ears of the Ormia ochracea. Appl. Phys. Lett. 2014, 105, 033701. [CrossRef]

6. Shen, Q.C.; Luo, Z.; Ma, S.; Tao, P.; Song, C.Y.; Wu, J.B.; Shang, W.; Deng, T. Bioinspired infrared sensing materials and systems. Adv. Mater. 2019, 30, 1707632. [CrossRef]

7. Scherzer, S.; Federle, W.; Al-Rasheid, K.A.S.; Hedrich, R. Venus flytrap trigger hairs are micronewton mechano-sensors that can detect small insect prey. Nat. Plants 2019, 5, 670-675. [CrossRef]

8. Jiang, Y.G.; Ma, Z.Q.; Zhang, D.Y. Flow field perception based on the fish lateral line system. Bioinspir. Biomimetics 2019, 14, 041001. [CrossRef]

9. Wang, K.J.; Zhang, J.Q.; Song, H.L.; Fang, Y.Q.; Wang, X.L.; Chen, D.B.; Liu, L.P.; Niu, S.C.; Yao, Z.W.; Han, Z.W.; et al. Highly efficient mechanoelectrical energy conversion based on the near-tip stress field of an antifracture slit observed in scorpions. Adv. Funct. Mater. 2019, 29, 1807693. [CrossRef]

10. Fratzl, P.; Barth, F.G. Biomaterial systems for mechanosensing and actuation. Nature 2009, 462, 442-448. [CrossRef]

11. Song, H.L.; Zhang, J.Q.; Chen, D.B.; Wang, K.J.; Niu, S.C.; Han, Z.W.; Ren, L.Q. Superfast and high-sensitivity printable strain sensors with bioinspired micron-scale cracks. Nanoscale 2017, 9, 1166-1173. [CrossRef]

12. Maschmann, M.R.; Ehlert, G.J.; Dickinson, B.T. Bioinspired carbon nanotube fuzzy fiber hair sensor for air-flow detection. Adv. Mater. 2014, 26, 3230-3234. [CrossRef] [PubMed]

13. Tee, B.C.K.; Chortos, A.; Berndt, A.; Nguyen, A.K.; Tom, A.; McGuire, A.; Mei, P.; Chou, H.-H.; Cui, B.; Deisseroth, K.; et al. A skin-inspired organic digital mechanoreceptor. Science 2015, 350, 313-316. [CrossRef] [PubMed]

14. Song, T.; Park, H.C.; Ahn, K.H. Proposal for high sensitivity force sensor inspired by auditory hair cells. Appl. Phys. Lett. 2009, 95, 013702. [CrossRef] 
15. Kang, D.; Pikhitsa, P.V.; Choi, Y.W.; Lee, C.; Shin, S.S.; Piao, L.; Park, B.; Suh, K.-Y.; Kim, T.; Choi, M. Ultrasensitive mechanical crack-based sensor inspired by the spider sensory system. Nature 2014, 516, 222-226. [CrossRef] [PubMed]

16. Schaber, C.F.; Gorb, S.N.; Barth, F.G. Force transformation in spider strain sensors: White light interferometry. J. R. Soc. Interface 2012, 9, 1254-1264. [CrossRef] [PubMed]

17. Hößl, B.; Böhm, H.J.; Schaber, C.F.; Rammerstorfer, F.G.; Barth, F.G. Finite element modeling of arachnid slit sensilla: II. Actual lyriform organs and the face deformations of the individual slits. J. Comp. Physiol. A. 2009, 195, 881-894. [CrossRef] [PubMed]

18. Krieg, M.; Dunn, A.R.; Goodman, M.B. Mechanical systems biology of C-elegans touch sensation. Bioessays 2015, 37, 335-344. [CrossRef]

19. Young, S.L.; Chyasnavichyus, M.; Erko, M.; Barth, F.G.; Fratzl, P.; Zlotnikov, I.; Politi, Y.; Tsukruk, V.V. A spider's biological vibration filter: Micromechanical characteristics of a biomaterial surface. Acta Biomater. 2014, 10, 4832-4842. [CrossRef]

20. Wang, K.J.; Zhang, J.Q.; Liu, L.P.; Chen, D.B.; Liu, L.; Han, Z.; Ren, L. Micro/nano-scale characterization and fatigue fracture resistance of mechanoreceptor with crack-shaped slit arrays in scorpion. J. Bionic Eng. 2019, 16, 410-422. [CrossRef]

21. Erko, M.; Younes-Metzler, O.; Rack, A.; Zaslansky, P.; Young, S.L.; Milliron, G.; Chyasnavichyus, M.; Barth, F.B.; Fratzl, P.; Tsukruk, V.; et al. Micro- and nano-structural details of a spider's filter for substrate vibrations: Relevance for low-frequency signal transmission. J. R. Soc. Interface 2015, 12, 20141111. [CrossRef] [PubMed]

22. Brownell, P.; Farley, R.D. Detection of vibrations in sand by tarsal sense organs of the nocturnal scorpion, Paruroctonus mesaensis. J. Comp. Physiol. 1979, 131, 23-30.

23. Bhaumik, S.K.; Sujata, M.; Venkataswamy, M.A. Fatigue failure of aircraft components. Eng. Fail. Anal. 2008, 15, 675-694. [CrossRef]

24. Vasco-Olmo, J.M.; James, M.N.; Christopher, C.J.; Patterson, E.A.; Díaz, F.A. Assessment of crack tip plastic zone size and shape and its influence on crack tip shielding. Fatigue Fract. Eng. Mater. Struct. 2016, 39, 969-981.

25. Oliver, W.C.; Pharr, G.M. Measurement of hardness and elastic modulus by instrumented indentation: Advances in understanding and refinements to methodology. J. Mater. Res. 2004, 19, 3-20. [CrossRef]

26. Meyers, M.A.; McKittrick, J.; Chen, P.Y. Structural Biological Materials: Critical Mechanics-Materials Connections. Science 2013, 339, 773-779.

27. Liu, X.X.; Dean, M.N.; Summers, A.P.; Earthman, J.C. Composite model of the shark's skeleton in bending: A novel architecture for biomimetic design of functional compression bias. Mater. Sci. Eng. C 2010, 30, 1077-1084.

28. Fratzl, P.; Kolednik, O.; Fischer, F.D.; Dean, M.N. The mechanics of tessellations-bioinspired strategies for fracture resistance. Chem. Soc. Rev. 2016, 45, 252-267.

29. Renshaw, J.; Holland, S.D.; Thompson, R.B. Measurement of crack opening stresses and crack closure stress profiles from heat generation in vibrating cracks. Appl. Phys. Lett. 2008, 93, 081914.

30. Topper, T.H.; Bonnen, J.J.F.; Khalil, M.; Farahani, A.V. The influence of biaxial strain ratio and strain range on crack growth mode and crack shape. J. Mater. Sci. 2010, 46, 215-227.

31. Pluvinage, G. (Ed.) Fracture and Fatigue Emanating from Stress Concentrators, 1st ed.; Springer: Dordrecht, The Netherlands, 2003.

Publisher's Note: MDPI stays neutral with regard to jurisdictional claims in published maps and institutional affiliations.

(C) 2020 by the authors. Licensee MDPI, Basel, Switzerland. This article is an open access article distributed under the terms and conditions of the Creative Commons Attribution (CC BY) license (http://creativecommons.org/licenses/by/4.0/). 